\title{
Metabolically Healthy Obesity Is Associated with an Increased Risk of Colorectal Adenoma Occurrence Diagnosed by Surveillance Colonoscopy
}

\author{
Hyunbeom Chae, Hyo-Joon Yang, Soo-Kyung Park, Yoon Suk Jung, Jung Ho Park, Dong II Park, \\ and Chong II Sohn \\ Division of Gastroenterology, Department of Internal Medicine and Gastrointestinal Cancer Center, Kangbuk Samsung Hospital, \\ Sungkyunkwan University School of Medicine, Seoul, Korea
}

\section{Article Info \\ Received March 6, 2020 \\ Revised April 30, 2020 \\ Accepted May 25, 2020 \\ Published online July 6, 2020 \\ Corresponding Author \\ Dong II Park \\ ORCID https://orcid.org/0000-0003-2307-8575 \\ E-mail diksmc.park@samsung.com}

Hyunbeom Chae and Hyo-Joon Yang

contributed equally to this work as first authors.

\begin{abstract}
Background/Aims: The risk of recurrence of colorectal adenoma among obese individuals without metabolic abnormalities or in those with metabolically healthy obesity is largely unexplored. Therefore, we longitudinally investigated the risk of adenoma occurrence in individuals undergoing surveillance colonoscopy according to metabolic status and obesity.

Methods: This retrospective cohort study included 16,872 Korean adults who underwent their first screening colonoscopy between 2003 and 2012 and who then underwent follow-up colonoscopy until 2017. Participants were categorized into a metabolically healthy nonobese group (reference group), a metabolically healthy obese group, a metabolically abnormal nonobese group, and a metabolically abnormal obese group. Hazard ratios (HRs) for adenoma recurrence compared to the reference group were calculated in each group.

Results: During a median follow-up duration of 47.3 months (interquartile range, 35.6 to 58.9 months), 3,673 (21.8\%) and $292(1.73 \%)$ participants developed adenoma and advanced adenoma, respectively. When age, sex, smoking, alcohol consumption, family history of colorectal cancer, and baseline adenoma risk were adjusted, the risk of adenoma recurrence was increased in metabolically healthy obese individuals ( $\mathrm{HR}, 1.33 ; 95 \%$ confidence interval [Cl], 1.12 to 1.57) and metabolically abnormal obese individuals (HR, 1.18; $95 \% \mathrm{Cl}, 1.08$ to 1.30 ) but not in metabolically abnormal nonobese individuals (HR, $1.03 ; 95 \% \mathrm{Cl}, 0.94$ to 1.13 ).

Conclusions: In this study, metabolically healthy obese individuals and metabolically abnormal obese individuals exhibited increased risks of occurrence of colorectal adenoma diagnosed by surveillance colonoscopy. This finding implies that obesity itself, even without metabolic abnormalities, is associated with an increased risk of adenoma recurrence. (Gut Liver 2021;15:383390)
\end{abstract}

Key Words: Colorectal neoplasm; Recurrence; Obesity, metabolically benign; Cohort studies; Colonoscopy

\section{INTRODUCTION}

Colorectal cancer (CRC) is the third most common cancer, and the second most common cause of cancer-related mortality worldwide, and over 1.8 million new cases of CRC and 0.8 million deaths were reported in $2018 .{ }^{1}$ Although there is a wide variation in the incidence of CRC among different countries, the global burden of this disease is expected to increase owing to the influences of dietary patterns, lifestyle factors, and obesity. On the contrary, as observed in developed countries, the mortality associated with CRC could decline with the increased application of CRC screening for early detection of cancer and removal of precancerous lesions. ${ }^{2}$

According to the current practice guidelines, surveillance interval after screening colonoscopy with or without polypectomy is determined solely based on the results of the previous colonoscopy. ${ }^{3}$ However, several studies have 
reported that the risk of occurrence of colorectal adenoma in surveillance colonoscopy also varies considerably according to age, ${ }^{4}$ sex, metabolic syndrome, ${ }^{5}$ obesity, ${ }^{6}$ and visceral obesity.

Obesity is a growing health concern that has global implications for increased risks of cancers, especially CRC. ${ }^{8}$ Most obese individuals usually have metabolic abnormalities. However, a small proportion of individuals retain a favorable metabolic profile; this is referred to as metabolically healthy obesity (MHO) phenotype. ${ }^{9}$ The clinical implications of $\mathrm{MHO}$ are still controversial. Previous studies have reported increased prevalence of colorectal neoplasm in individuals with MHO. ${ }^{10,11}$ On the contrary, in another study, the increased risk of prevalent advanced neoplasm was evident only in obese individuals with metabolic abnormalities but not in those with MHO phenotype. ${ }^{12}$ Moreover, no study has evaluated the association between $\mathrm{MHO}$ and the risk of adenoma occurrence in surveillance colonoscopy.

Therefore, in this study, we longitudinally investigated the risk of adenoma occurrence in surveillance colonoscopy according to metabolic status and obesity in a large cohort of Korean individuals.

\section{MATERIALS AND METHODS}

\section{Study population}

This retrospective cohort study included asymptomatic men and women who underwent their first screening colonoscopy, defined as index colonoscopy, between January 2003 and December 2012 and had at least one follow-up colonoscopy until June 2017 at Kangbuk Samsung Hospital, Seoul, Korea. As previously described, ${ }^{12}$ individuals who had undergone previous colorectal examination, those with a history of CRC or colorectal surgery, those with a history of inflammatory bowel disease, those who underwent incomplete index colonoscopy, and those with missing data were excluded at baseline. In the present study, participants with a diagnosis of CRC at index colonoscopy, those who underwent colorectal surgery during follow-up, those with a new diagnosis of inflammatory bowel disease, those who underwent incomplete surveillance colonoscopy, those with missing surveillance data, and those who were followed up for less than 1 year were further excluded.

The study participants were categorized into four groups according to their metabolic status and obesity, as follows: (1) metabolically healthy nonobese (MHNO) group: individuals with no metabolic abnormality who were not obese; (2) MHO group: individuals without any metabolic abnormality who were obese; (3) metabolically abnormal nonobese (MANO) group: individuals with metabolic abnormalities who were not obese; and (4) metabolically abnormal obese (MAO) group: individuals with metabolic abnormalities who were obese. This study was approved by the Institutional Review Board of Kangbuk Samsung Hospital (IRB number: KBSMC 2017-07-024). The requirement for obtaining informed consent from the participants was waived.

\section{Definitions and measurements}

Metabolic abnormality was defined as the presence of abdominal obesity (waist circumference $\geq 90 \mathrm{~cm}$ for men and $\geq 85 \mathrm{~cm}$ for women), elevated triglycerides $(\geq 150 \mathrm{mg} /$ $\mathrm{dL})$, increased low high-density lipoprotein cholesterol $(<40$ $\mathrm{mg} / \mathrm{dL}$ for men and $<50 \mathrm{mg} / \mathrm{dL}$ for women), high blood pressure $(\geq 130 / 85 \mathrm{~mm} \mathrm{Hg}$ or use of antihypertensive medications), or impaired fasting glucose (fasting plasma glucose $\geq 100 \mathrm{mg} / \mathrm{dL}$, hemoglobin Alc $\geq 6.5 \%$, or use of diabetes medication). A Korean-specific criterion was adopted for the definition of abdominal obesity, ${ }^{13}$ while the others were defined based on the modified National Cholesterol Education Program Adult Treatment Panel III criteria. ${ }^{14}$ Obesity was also defined according to the Asianspecific criteria, ${ }^{15}$ as body mass index (BMI) $\geq 25 \mathrm{~kg} / \mathrm{m}^{2}$.

A standardized self-administered questionnaire was used to collect information regarding the demographic and clinical characteristics of the patients, including medication use, health behaviors, and family history of CRC at baseline. Smoking status was categorized as never, former, and current smokers according to the definitions from the U.S. Centers for Disease Control (https://www.cdc. gov/). ${ }^{16}$ Never smoker was defined as an individual who has smoked less than 100 cigarettes per lifetime. Former smoker was defined as an individual who has smoked at least 100 cigarettes but quit smoking. Current smoker was defined as an individual who is currently smoking and has smoked 100 or more cigarettes in his or her lifetime. Alcohol consumption $\geq 4$ times per week was considered as heavy drinking. Moderate to vigorous exercise $\geq 1$ times per week was defined as regular exercise. A history of CRC in $\geq 1$ first-degree relatives regardless of age was considered as a family history of CRC. The weight, height, waist circumference, and sitting blood pressure of the participants were measured by trained nurses. Blood samples were taken after at least 10 hours of fasting.

\section{Colonoscopy and histologic examination}

All colonoscopic examinations were performed by 13 board-certified colonoscopists. The participants were administered $4 \mathrm{~L}$ of polyethylene glycol lavage solution 
for bowel cleansing. All suspected neoplastic lesions were removed via biopsy forceps, snare polypectomy, or endoscopic mucosal resection. The removed specimens were histologically assessed by experienced gastrointestinal pathologists. Baseline colonoscopy findings were categorized into no adenoma, low-risk adenoma, and high-risk adenoma. Low-risk adenoma was defined as 1-2 tubular adenomas measuring $<10 \mathrm{~mm}$ in size, and high-risk adenoma was defined as advanced adenomas or patients with $\geq 3$ adenomas. ${ }^{3}$ Advanced adenoma was defined by the presence of one of the following features: a lesion measuring $\geq 10 \mathrm{~mm}$ in diameter, showing a tubulovillous or villous structure, or high-grade dysplasia. The findings from colonoscopies conducted within 1 year from the index colonoscopy were considered as a part of index colonoscopy findings.

\section{Statistical analysis}

The chi-square test, linear-by-linear association, or Fisher exact test was used to compare categorical variables, and one-way analysis of variance or Kruskal-Wallis test was used to compare continuous variables between the groups. The cumulative risk of adenoma recurrence in each group was also estimated using the Kaplan-Meier method, with differences determined using the log-rank test. The risk of adenoma recurrence on surveillance colonoscopy associated with metabolic abnormality and obesity was assessed using the Cox proportional hazard regression model by computing the hazard ratio (HR) and corresponding 95\% confidence interval (CI) after adjusting for major confounding factors including age, sex, smoking status, alcohol consumption, family history of CRC, regular exercise, and baseline colonoscopy findings. All reported p-values were two tailed, and a p-value $<0.05$ was considered statistically significant. STATA, version 15.0 (StataCorp LP, College Station, TX, USA) was used for statistical analyses.

\section{RESULTS}

\section{Baseline characteristics of the study population}

Among 70,336 participants who had undergone index colonoscopy from 2003 and 2012, a total of 19,734 individuals underwent follow-up colonoscopy until 2017. In total, 2,862 participants were excluded because of the following reasons: CRC or colorectal surgery during follow-up $(n=35)$, new diagnosis of inflammatory bowel disease during follow-up ( $\mathrm{n}=8$ ), incomplete surveillance colonoscopy $(\mathrm{n}=1,897)$, missing data $(\mathrm{n}=122)$, and follow-up duration $<1$ year $(\mathrm{n}=819)$. Some participants satisfied more than one exclusion criteria. Consequently, 16,872 participants were included in the analysis and categorized into MHNO $(\mathrm{n}=4,157 ; 24.6 \%)$, MHO $(\mathrm{n}=698 ; 4.1 \%)$, MANO $(\mathrm{n}=6,272$; $37.2 \%)$, and MAO groups ( $\mathrm{n}=5,745 ; 34.1 \%$ ) (Fig. 1). The included participants were more likely to be men and to have advanced adenoma or cancer in index colonoscopy than those who were not included (Supplementary Table 1). However, there were no clinically meaningful differences in metabolic status and obesity between the two groups of individuals although the p-values were statistically significant because of large sample size.

The baseline patient characteristics and index colonoscopy findings are described in Table 1 . The mean age ( \pm standard deviation) of the overall participants was $42.0 \pm 7.1$ years, and $80.4 \%$ of the participants $(13,659 / 16,872)$ were men. The participants in MAO and MANO groups were older (aged 42.1 and 42.8 years, respectively), and those in MAO and MHO groups were more likely to be men (89.8\% and $91.6 \%$, respectively) (all $\mathrm{p}<0.001)$. As expected, BMI, lifestyle factors, and metabolic parameters showed significant differences between the groups. In the index colonoscopy, MAO group had the highest prevalence of high- and low-risk adenomas (6.1\% and $21.7 \%$ ), followed by MANO (5.3\% and 19.8\%), MHO

Potential participants who underwent screening colonoscopy at Kangbuk Samsung Hospital from 2003 to $2012(n=121,794)$

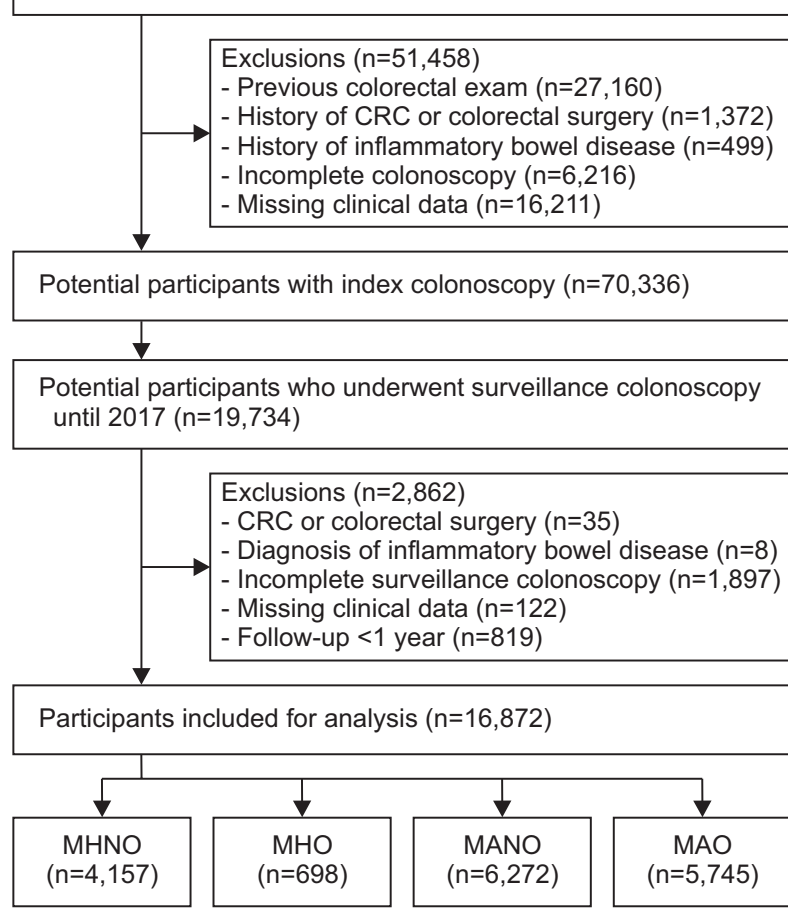

Fig. 1. Flowchart of study participants.

CRC, colorectal cancer; MHNO, metabolically healthy nonobese; $\mathrm{MHO}$, metabolically healthy obesity; MANO, metabolically abnormal nonobese; MAO, metabolically abnormal obese. 
Table 1. Baseline Characteristics of Study Participants

\begin{tabular}{|c|c|c|c|c|c|c|}
\hline Characteristics & Overall ( $n=16,872$ ) & MHNO $(n=4,157)$ & $\mathrm{MHO}(\mathrm{n}=698)$ & MANO $(n=6,272)$ & MAO $(n=5,745)$ & $\mathrm{p}$-value \\
\hline Age, yr & $42.0 \pm 7.1$ & $40.7 \pm 6.6$ & $40.7 \pm 6.9$ & $42.8 \pm 7.2$ & $42.1 \pm 7.0$ & $<0.001$ \\
\hline Sex & & & & & & $<0.001$ \\
\hline Men & $13,569(80.4)$ & $2,876(69.2)$ & $639(91.6)$ & $4,898(78.1)$ & $5,156(89.8)$ & \\
\hline Women & $3,303(19.6)$ & $1,281(30.8)$ & $59(8.5)$ & $1,374(21.9)$ & 589 (10.3) & \\
\hline $\mathrm{BMI}, \mathrm{kg} / \mathrm{m}^{2}$ & $24.3 \pm 3.0$ & $22.1 \pm 1.9$ & $25.8 \pm 0.9$ & $23.0 \pm 2.1$ & $27.1 \pm 2.4$ & $<0.001$ \\
\hline \multicolumn{7}{|l|}{ Waist circumference, $\mathrm{cm}$} \\
\hline Men & $86.0 \pm 7.4$ & $80.5 \pm 5.1$ & $86.4 \pm 2.6$ & $83.0 \pm 5.7$ & $91.9 \pm 6.2$ & $<0.001$ \\
\hline Women & $77.6 \pm 7.8$ & $74.2 \pm 5.5$ & $80.9 \pm 2.9$ & $76.7 \pm 6.6$ & $86.7 \pm 8.1$ & $<0.001$ \\
\hline Smoking & & & & & & $<0.001$ \\
\hline Never & $9,520(56.4)$ & $2,574(61.9)$ & 429 (61.5) & $3,436(54.8)$ & $3,081(53.6)$ & \\
\hline Former & $2,349(13.9)$ & $516(12.4)$ & 86 (12.3) & $905(14.4)$ & $842(14.7)$ & \\
\hline Current & $5,003(29.7)$ & $1,067(25.7)$ & $183(26.2)$ & $1,931(30.8)$ & $1,822(31.7)$ & \\
\hline Alcohol intake & & & & & & 0.005 \\
\hline No & $2,682(15.9)$ & $714(17.2)$ & $116(16.6)$ & 971 (15.5) & 881 (15.3) & \\
\hline Moderate & $13,678(81.1)$ & 3,334 (80.2) & $573(82.1)$ & $5,098(81.3)$ & $4,673(81.3)$ & \\
\hline Heavy & $512(3.0)$ & $109(2.6)$ & $9(1.3)$ & 203 (3.2) & 191 (3.3) & \\
\hline CRC family history & $868(5.1)$ & $186(4.5)$ & $21(3.0)$ & $374(6.0)$ & $287(5.0)$ & $<0.001$ \\
\hline Systolic BP, mm Hg & $114.8 \pm 12.7$ & $107.6 \pm 9.6$ & $108.8 \pm 9.4$ & $118.4 \pm 12.7$ & $116.7 \pm 12.7$ & $<0.001$ \\
\hline Diastolic BP, mm Hg & $73.6 \pm 9.4$ & $67.5 \pm 6.1$ & $68.0 \pm 5.6$ & $76.8 \pm 9.2$ & $75.3 \pm 9.5$ & $<0.001$ \\
\hline Hypertension & $2,736(16.2)$ & - & - & $1,536(24.5)$ & $1,200(20.9)$ & $<0.001$ \\
\hline Diabetes & $921(5.5)$ & - & - & $460(7.3)$ & $461(8.0)$ & $<0.001$ \\
\hline Regular exercise & $8,906(52.8)$ & $2,253(54.2)$ & 365 (52.3) & $3,222(51.4)$ & $3,066(53.4)$ & 0.027 \\
\hline Fasting glucose, mg/dL & $93.9 \pm 15.2$ & $87.7 \pm 7.2$ & $88.9 \pm 7.0$ & $95.4 \pm 16.8$ & $97.2 \pm 16.8$ & $<0.001$ \\
\hline Insulin, $\mu \mathrm{IU} / \mathrm{mL}$ & $4.9(3.0-7.4)$ & $3.2(2.1-5.3)$ & $4.5(3.0-6.8)$ & $4.8(3.0-7.2)$ & $6.4(4.2-8.9)$ & $<0.001$ \\
\hline $\mathrm{HbA} 1 \mathrm{c}, \%$ & $5.6(5.4-5.8)$ & $5.5(5.4-5.7)$ & $5.5(5.4-5.7)$ & $5.6(5.4-5.8)$ & $5.7(5.5-5.9)$ & $<0.001$ \\
\hline HOMA-IR & $1.1(0.7-1.7)$ & $0.7(0.4-1.2)$ & $1.0(0.6-1.5)$ & $1.1(0.7-1.7)$ & $1.5(1.0-2.1)$ & $<0.001$ \\
\hline Total cholesterol, mg/dL & $202.4 \pm 34.4$ & $194.1 \pm 31.4$ & $202.3 \pm 31.2$ & $201.8 \pm 34.5$ & $209.0 \pm 35.3$ & $<0.001$ \\
\hline Triglyceride, mg/dL & 104 (73-151) & 76 (58-99) & $91(70-116)$ & 107 (75-159) & $136(96-191)$ & $<0.001$ \\
\hline HDL-C, mg/dL & $53.7 \pm 13.0$ & $60.4 \pm 12.6$ & $54.4 \pm 10.2$ & $53.8 \pm 13.4$ & $48.7 \pm 10.7$ & $<0.001$ \\
\hline $\mathrm{LDL}-\mathrm{C}, \mathrm{mg} / \mathrm{dL}$ & $126.6 \pm 31.6$ & $118.6 \pm 29.4$ & $130.2 \pm 29.9$ & $125.3 \pm 31.3$ & $133.4 \pm 32.2$ & $<0.001$ \\
\hline Index colonoscopy & & & & & & $<0.001$ \\
\hline No adenoma & $12,606(74.7)$ & $3,219(77.4)$ & 536 (76.8) & $4,700(74.9)$ & 4,151 (72.3) & \\
\hline Low-risk adenoma & $3,370(20.0)$ & 755 (18.2) & 132 (18.9) & $1,239(19.8)$ & $1,244(21.7)$ & \\
\hline High-risk adenoma & $896(5.3)$ & $183(4.4)$ & $30(4.3)$ & 333 (5.3) & $350(6.1)$ & \\
\hline
\end{tabular}

Data are presented as mean \pm SD, number (\%), or median (interquartile range).

MHNO, metabolically healthy nonobese; MHO, metabolically healthy obesity; MANO, metabolically abnormal nonobese; MAO, metabolically abnormal obese; BMI, body mass index; CRC, colorectal cancer; BP, blood pressure; HbA1c, hemoglobin A1c; HOMA-IR, homeostatic model assessment-insulin resistance; HDL-C, high density lipoprotein-cholesterol; LDL-C, low density lipoprotein-cholesterol.

(4.3\% and $18.9 \%$ ), and MHNO groups (4.4\% and $18.2 \%$, respectively) $(\mathrm{p}<0.001)$.

\section{Cumulative risk of colorectal adenoma in surveillance colonoscopy}

During a median follow-up duration of 47.3 months (interquartile range, 35.6 to 58.9 months), 3,673 (21.8\%) and $292(1.73 \%)$ participants developed adenoma and advanced adenoma, as observed in surveillance colonoscopy, respectively. No participant was diagnosed with CRC in the follow-up examination.

The cumulative risk of developing adenoma was higher in $\mathrm{MHO}$ and MAO groups than in MANO and MHNO groups (log-rank $\mathrm{p}<0.001$ ) (Fig. $2 \mathrm{~A}$ ). The 3 - and 5 -year cumulative risks for adenoma occurrence were $8.0 \%$ and
$36.0 \%$ in $\mathrm{MHO}$ group and $9.0 \%$ and $32.9 \%$ in $\mathrm{MAO}$ group, respectively, whereas the risks were $6.5 \%$ and $27.5 \%$ in MANO group and $5.7 \%$ and $25.3 \%$ in MHNO group, respectively (Table 2). The risk of developing advanced adenoma also seemed higher in $\mathrm{MHO}$ and MAO groups than in MANO and MHNO groups; however, the difference was only marginally significant because of the small number of advanced lesions ( $\mathrm{p}=0.062$ ) (Table 2, Fig. 2B).

\section{Adjusted hazards ratios for colorectal adenoma according to metabolic status and obesity}

Compared with MHNO group as a reference, all the other groups showed significantly increased HRs for developing adenoma in surveillance colonoscopy $(\mathrm{p}<0.001)$ (Table 3). The multiple Cox regression model was con- 
A

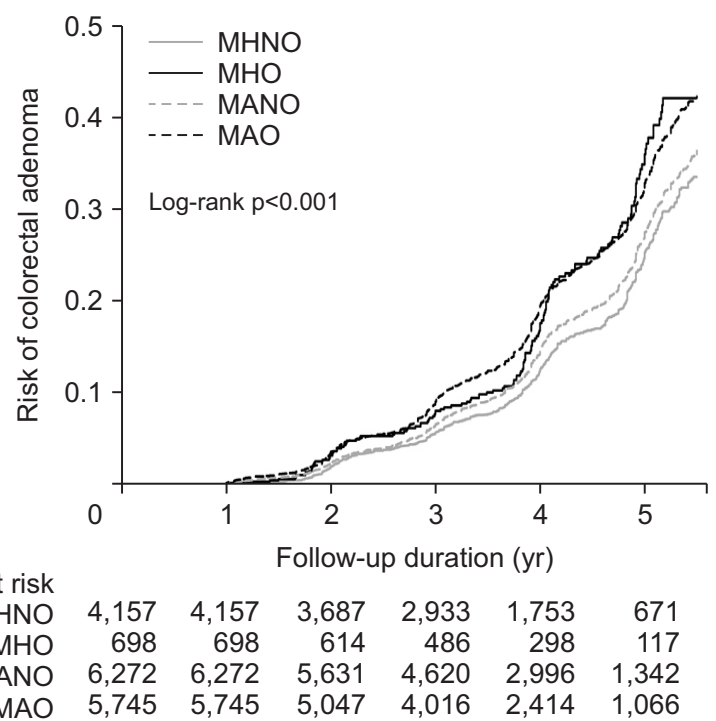

B

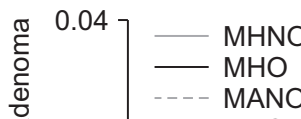

$0.03-\ldots$

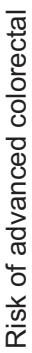

No. at risk

$\begin{array}{lllllll}\text { MHNO } & 4,157 & 4,157 & 3,716 & 2,995 & 1,809 & 709\end{array}$

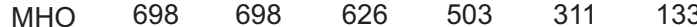

$\begin{array}{lllllll}\text { MANO } & 6,272 & 6,272 & 5,704 & 4,763 & 3,157 & 1,487\end{array}$

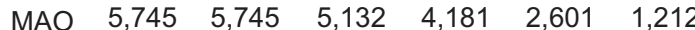

Fig. 2. Cumulative incidence of (A) colorectal adenoma and (B) advanced colorectal adenoma among four groups according to metabolic status and obesity.

MHNO, metabolically healthy nonobese; MHO, metabolically healthy obesity; MANO, metabolically abnormal nonobese; MAO, metabolically abnormal obese.

Table 2. Cumulative Risk of Adenoma and Advanced Adenoma Occurrence Diagnosed by Surveillance Colonoscopy among the Four Patient Groups According to Metabolic Status and Obesity

\begin{tabular}{lccccc}
\hline \multicolumn{1}{c}{ Outcome } & Overall & MHNO & MHO & MANO & MAO \\
\hline Any adenoma & & & & & \\
$3 \mathrm{yr}$ & $7.2(6.8-7.7)$ & $5.7(5.0-6.5)$ & $8.0(6.1-10.5)$ & $6.5(5.9-7.2)$ & $9.0(8.3-9.9)$ \\
$5 \mathrm{yr}$ & $29.2(28.2-30.2)$ & $25.3(23.3-27.5)$ & $36.0(30.8-41.8)$ & $27.5(26.0-29.1)$ & $32.9(31.2-34.7)$ \\
Advanced adenoma & & & & & \\
$3 \mathrm{yr}$ & $0.53(0.42-0.66)$ & $0.40(0.24-0.67)$ & $0.47(0.15-1.46)$ & $0.44(0.29-0.66)$ & $0.72(0.52-1.00)$ \\
$5 \mathrm{yr}$ & $2.15(1.85-2.51)$ & $2.28(1.62-3.22)$ & $2.12(1.01-4.40)$ & $1.76(1.35-2.29)$ & $2.57(2.03-3.25)$ \\
\hline
\end{tabular}

Data are presented as \% (95\% confidence interval).

MHNO, metabolically healthy nonobese; MHO, metabolically healthy obesity; MANO, metabolically abnormal nonobese; MAO, metabolically abnormal obese.

structed after adjusting for age, sex, smoking status, alcohol consumption, family history of CRC, regular exercise, and baseline risk of adenoma. In this model, HRs for adenoma development were still significantly increased in MHO group (HR, 1.33; 95\% CI, 1.12 to 1.57 ) and MAO group (HR, 1.18; 95\% CI, 1.08 to 1.30 ) but not in MANO group (HR, 1.03; 95\% CI, 0.94 to 1.13). The adjusted HR for developing advanced adenoma was also increased in MHO (HR, 1.31; 95\% CI, 0.71 to 2.42) and MAO (HR, 1.15; $95 \% \mathrm{CI}, 0.82$ to 1.61 ) groups compared with $\mathrm{MHNO}$ group; however, the difference did not reach statistical significance owing to the wide range of the estimates.

\section{Number of adenomas according to metabolic status and obesity}

As ad hoc analyses, we additionally investigated the risks of developing 3 or more and 5 or more adenomas according to metabolic status and obesity. In the results, the cumulative risks of developing 3 or more and 5 or more adenomas were significantly increased only in the MAO group than in the other groups (log-rank $\mathrm{p}<0.001$ and $\mathrm{p}=0.010$, respectively) (Supplementary Fig. 1). In the adjusted Cox regression models, the MAO group was associated with significantly increased risk of developing 3 or more adenomas than the other groups (HR, 1.51; 95\% CI, 1.14 to 2.00) (Supplementary Table 2).

\section{DISCUSSION}

In this large cohort study of Korean adults who underwent follow-up colonoscopy, compared with normal weight, obesity without metabolic abnormality and obesity with metabolic abnormality were associated with high 
Table 3. Hazard Ratios for Occurrence of Adenoma and Advanced Adenoma Diagnosed by Surveillance Colonoscopy among the Four Patient Groups According to Metabolic Status and Obesity

\begin{tabular}{|c|c|c|c|c|c|c|c|}
\hline Outcome & Person-years & Incident case & $\begin{array}{c}\text { Incidence rate } \\
\text { (per 1,000 person-years) }\end{array}$ & $\begin{array}{l}\text { Crude HR } \\
(95 \% \mathrm{Cl})\end{array}$ & $p$-value & $\begin{array}{l}\text { Adjusted HR* } \\
\text { (95\% Cl) }\end{array}$ & $p$-value \\
\hline \multicolumn{8}{|l|}{ Any adenoma } \\
\hline MHNO & 15,750 & 680 & 43.2 & 1 (reference) & & 1 (reference) & \\
\hline $\mathrm{MHO}$ & 2,626 & 162 & 61.7 & $1.45(1.22-1.72)$ & $<0.001$ & $1.33(1.12-1.57)$ & 0.001 \\
\hline MANO & 24,962 & 1,382 & 55.4 & $1.15(1.05-1.26)$ & 0.003 & $1.03(0.94-1.13)$ & 0.494 \\
\hline MAO & 22,013 & 1,449 & 65.8 & $1.43(1.30-1.56)$ & $<0.001$ & $1.18(1.08-1.30)$ & $<0.001$ \\
\hline p overall & & & & & $<0.001$ & & $<0.001$ \\
\hline \multicolumn{8}{|c|}{ Advanced adenoma } \\
\hline MHNO & 15,997 & 50 & 3.1 & 1 (reference) & & 1 (reference) & \\
\hline $\mathrm{MHO}$ & 2,709 & 13 & 4.8 & $1.53(0.83-2.81)$ & 0.175 & $1.31(0.71-2.42)$ & 0.393 \\
\hline MANO & 25,752 & 108 & 4.2 & $1.12(0.80-1.57)$ & 0.497 & $0.89(0.63-1.25)$ & 0.511 \\
\hline MAO & 22,829 & 121 & 5.3 & $1.46(1.05-2.04)$ & 0.024 & $1.15(0.82-1.61)$ & 0.427 \\
\hline p overall & & & & & 0.065 & & 0.233 \\
\hline
\end{tabular}

$\mathrm{HR}$, hazard ratio; $\mathrm{Cl}$, confidence interval; MHNO, metabolically healthy nonobese; MHO, metabolically healthy obesity; MANO, metabolically abnormal nonobese; MAO, metabolically abnormal obese.

*Adjusted for age, sex, smoking status, alcohol consumption, family history of colorectal cancer, regular exercise, and baseline risk of adenoma.

risks of incident colorectal adenoma, over a 5-year surveillance period. The risk of incident advanced adenoma in these populations also increased with an extent similar to that of overall adenoma, suggesting possible increased risk of advanced adenoma in individuals with $\mathrm{MHO}$ although the differences between the groups were not statistically significant.

In our study, compared with patients from $\mathrm{MHNO}$ group, those from $\mathrm{MHO}$ and $\mathrm{MAO}$ groups were at increased risks of adenoma occurrence, with HRs of 1.33 (95\% CI, 1.12 to 15.7 ) and 1.18 (95\% CI, 1.08 to 1.30 ), respectively. In previous studies, the effects of obesity and metabolic syndrome on adenoma recurrence were individually assessed. A pooled analysis of 8,213 participants from seven prospective studies showed a statistically significant association between BMI and the risk of adenoma at follow-up colonoscopy $(\mathrm{p}<0.001) .{ }^{17}$ Recently, Im et al. ${ }^{7}$ examined the association between visceral obesity and the occurrence of colorectal adenoma in surveillance colonoscopy. In this study, increasing BMI as well as visceral adiposity and waist circumference were associated with the risk of incident adenoma (HR, 1.33; 95\% CI, 1.18 to 1.46). Meanwhile, Chiu et al. ${ }^{5}$ showed that metabolic syndrome was associated with the detection of advanced neoplasms in surveillance colonoscopy after negative or low-risk index colonoscopy (HR, 2.07; 95\% CI, 1.13 to 3.81 and HR, 2.34; $95 \mathrm{CI}, 1.01$ to 5.41 ; respectively). Another study reported metabolic syndrome as an independent risk factor of adenoma occurrence in follow-up colonoscopy (HR, 1.28; 95\% CI, 1.09 to 1.51$).{ }^{18}$ However, the influence of MHO has been evaluated only for prevalent colorectal neoplasm in a cross-sectional design. Individuals with $\mathrm{MHO}$ were associated with increased risk of prevalent adenoma com- pared with metabolically healthy normal-weight individuals, with ORs of 1.44 (95\% CI, 1.23 to 1.69) and 1.25 (95\% CI, 1.09 to 1.43 ), respectively, in two previous studies, ${ }^{10,11}$ but not with advanced neoplasm (odds ratio, 0.99; 95\% CI, 0.67 to 1.46 ), in another study. ${ }^{12}$ In the current study, the association between $\mathrm{MHO}$ and the risk of adenoma occurrence was evaluated in surveillance colonoscopy in 16,872 individuals who underwent a median of 47.3 months of follow-up, which is a strength of this study. Our findings are consistent with those of previous studies on obesity and adenoma recurrence, ${ }^{6,717}$ and on $\mathrm{MHO}$ and prevalent adenoma. ${ }^{10,11}$ Further, our results provide new evidence suggesting that excessive body weight, even in the absence of metabolic abnormalities, increases the risk of colorectal adenoma occurrence in surveillance colonoscopy, not only in initial screening colonoscopy.

The mechanisms that link obesity and adenoma recurrence are still unclear. Among them, insulin would be the best-established biochemical link. ${ }^{19-21}$ Obesity and metabolic syndrome cause insulin resistance and hyperinsulinemia, and increased blood insulin levels lower the levels of insulin-like growth factor-binding protein, leading to increased levels of free insulin-like growth factor-1. Increased insulin-like growth factor-1 stimulates the proliferation of colonic cells and inhibits apoptosis through various intracellular signal transduction processes. In addition, adipokines such as leptin and adiponectin secreted from adipocytes and cytokines such as tumor necrosis factor- $\alpha$, interleukin-6, interleukin-8, and interleukin-10 are known to promote cancer development by promoting cell proliferation and angiogenesis. ${ }^{22-25}$

Notably, the HR in MHO group for advanced adenoma recurrence (1.31; $95 \% \mathrm{CI}, 0.71$ to 2.42$)$ was quite similar 
to that for overall adenoma (1.33; 95\% CI, 1.12 to 1.57$)$. A similar finding was observed for MAO group (HR, 1.15; 95\% CI, 0.82 to 1.61 for advanced adenoma and HR, 1.18; 95\% CI, 1.08 to 1.30 for overall adenoma). These findings may indicate that $\mathrm{MHO}$ is possibly associated with increased risk of advanced adenoma occurrence in surveillance colonoscopy. However, these results were not statistically significant with very wide confidence ranges owing to the small number of events. Meanwhile, the risk of developing increased number of adenomas was increased in the MAO group but not in the MHO group. These results suggest that the mechanism underpinning the development of many adenomas may be different from those for the development of overall or advanced adenoma. Therefore, further evidences are required to elucidate whether the risks of developing advanced adenoma and many adenomas in surveillance colonoscopy are increased in individuals with MHO.

Our results may not necessitate different surveillance intervals for adults with $\mathrm{MHO}$ or $\mathrm{MAO}$, compared with normal-weight individuals. The prevalence of incident adenoma after 5 years of follow-up ranged between $25.3 \%$ and $36.0 \%$. It is noteworthy that the risk of advanced adenoma was not significantly different among the groups, although the possibility of difference has been mentioned. Therefore, it may be advisable to maintain better adherence in post-colonoscopy surveillance and conduct detailed inspection during follow-up colonoscopy for obese adults irrespective of the presence or absence of metabolic abnormalities.

Our study has multiple strengths. First, we adopted a well-established cohort as a baseline population that was involved in several studies. ${ }^{12,26}$ Second, as previously mentioned, this study involved 16,872 individuals who underwent surveillance colonoscopy, with a long follow-up duration of a median of 47.3 months. Third, multiple confounders were included in the adjusted analysis, including lifestyle factors such as smoking, alcohol consumption, and regular exercise. However, this study also has several inherent limitations. First, this was a retrospective cohort study, with most participants being employees who underwent colonoscopies for a health check-up program. Thus, some degree of selection bias may have occurred. Second, although the number of participants was large, a relatively small proportion $(24.0 \% ; n=16,872 / 70,336)$ of participants who underwent initial colonoscopy returned for surveillance exam. Because of higher proportion of men and advanced adenoma in the included participants than in those who were not included, the estimated risks of overall and advanced adenoma in surveillance colonoscopy might have been overestimated in the overall cohort. However, the association between obesity with or without metabolic abnormality and the risk of incident colorectal adenoma might not have affected by the large proportion of lost to follow-up because follow-up loss were not associated with these parameters. Third, we could not evaluate cytokines, such as insulin-like growth factor-1 and adipokines, which are considered to have an important role in the association between obesity and adenoma recurrence.

In conclusion, in this large cohort of participants who underwent colonoscopy follow-up, MHO individuals and MAO individuals exhibited increased risks of colorectal adenoma occurrence in surveillance colonoscopy. This implies that obesity itself, even in the absence of metabolic abnormalities, is associated with an increased risk of adenoma recurrence.

\section{CONFLICTS OF INTEREST}

No potential conflict of interest relevant to this article was reported.

\section{AUTHOR CONTRIBUTIONS}

Conceptualization: D.I.P. Data curation: H.C., H.J.Y. Formal analysis: H.J.Y. Methodology: H.J.Y., S.K.P., Y.S.J., J.H.P., D.I.P., C.I.S. Project administration: D.I.P. Visualization: H.J.Y. Writing - original draft: H.C. Writing - review \& editing: H.J.Y., D.I.P. Approval of final manuscript: all authors.

\section{ORCID}

Hyunbeom Chaehttps://orcid.org/0000-0001-6721-2479 Hyo-Joon Yang https://orcid.org/0000-0002-0265-672X Soo-Kyung Park https://orcid.org/0000-0001-8822-9632 Yoon Suk Jung https://orcid.org/0000-0002-1963-7170 Jung Ho Park https://orcid.org/0000-0002-8367-4371 Dong Il Park https://orcid.org/0000-0003-2307-8575 Chong Il Sohn https://orcid.org/0000-0002-9748-8537

\section{REFERENCES}

1. Bray F, Ferlay J, Soerjomataram I, Siegel RL, Torre LA, Jemal A. Global cancer statistics 2018: GLOBOCAN estimates of incidence and mortality worldwide for 36 cancers in 185 countries. CA Cancer J Clin 2018;68:394-424.

2. Arnold M, Sierra MS, Laversanne M, Soerjomataram I, Je- 
mal A, Bray F. Global patterns and trends in colorectal cancer incidence and mortality. Gut 2017;66:683-691.

3. Lieberman DA, Rex DK, Winawer SJ, Giardiello FM, Johnson DA, Levin TR. Guidelines for colonoscopy surveillance after screening and polypectomy: a consensus update by the US Multi-Society Task Force on Colorectal Cancer. Gastroenterology 2012;143:844-857.

4. Pommergaard HC, Burcharth J, Rosenberg J, Raskov H. Advanced age is a risk factor for proximal adenoma recurrence following colonoscopy and polypectomy. Br J Surg 2016;103:e100-e105.

5. Chiu HM, Lee YC, Tu CH, et al. Effects of metabolic syndrome and findings from baseline colonoscopies on occurrence of colorectal neoplasms. Clin Gastroenterol Hepatol 2015;13:1134-1142.

6. Jung YS, Park JH, Park DI, Sohn CI, Choi K. Weight change and obesity are associated with a risk of adenoma recurrence. Dig Dis Sci 2016;61:2694-2703.

7. Im JP, Kim D, Chung SJ, et al. Visceral obesity as a risk factor for colorectal adenoma occurrence in surveillance colonoscopy. Gastrointest Endosc 2018;88:119-127.

8. Finucane MM, Stevens GA, Cowan MJ, et al. National, regional, and global trends in body-mass index since 1980: systematic analysis of health examination surveys and epidemiological studies with 960 country-years and 9.1 million participants. Lancet 2011;377:557-567.

9. Chang Y, Jung HS, Yun KE, et al. Metabolically healthy obesity is associated with an increased risk of diabetes independently of nonalcoholic fatty liver disease. Obesity (Silver Spring) 2016;24:1996-2003.

10. Sinn DH, Min YW, Son HJ, et al. Metabolically-healthy obesity is associated with higher prevalence of colorectal adenoma. PLoS One 2017;12:e0179480.

11. Yun KE, Chang Y, Jung HS, et al. Impact of body mass index on the risk of colorectal adenoma in a metabolically healthy population. Cancer Res 2013;73:4020-4027.

12. Kim JY, Park DI, Yu J, et al. Increased risk of advanced colorectal neoplasia among Korean men with metabolic abnormality and obesity. Clin Gastroenterol Hepatol 2016;14:1310-1316.

13. Lee SY, Park HS, Kim DJ, et al. Appropriate waist circumference cutoff points for central obesity in Korean adults. Diabetes Res Clin Pract 2007;75:72-80.

14. Alberti KG, Eckel RH, Grundy SM, et al. Harmonizing the metabolic syndrome: a joint interim statement of the International Diabetes Federation Task Force on Epidemiology and Prevention; National Heart, Lung, and Blood Institute; American Heart Association; World Heart Federation; International Atherosclerosis Society; and International Association for the Study of Obesity. Circulation 2009;120:16401645.

15. Wen CP, David Cheng TY, Tsai SP, et al. Are Asians at greater mortality risks for being overweight than Caucasians? Redefining obesity for Asians. Public Health Nutr 2009;12:497506.

16. Centers for Disease Control and Prevention (CDC). Adult tobacco use information [Internet]. Hyattsville: CDC; c2017 [cited 2020 Jun 23]. Available from: https://www.cdc.gov/ nchs/nhis/tobacco/tobacco_glossary.htm.

17. Jacobs ET, Ahnen DJ, Ashbeck EL, et al. Association between body mass index and colorectal neoplasia at follow-up colonoscopy: a pooling study. Am J Epidemiol 2009;169:657-666.

18. Kim NH, Park JH, Park DI, Sohn CI, Choi K, Jung YS. Metabolic syndrome is a risk factor for adenoma occurrence at surveillance colonoscopy: a single-center experience in Korea. Medicine (Baltimore) 2016;95:e4454.

19. Frezza EE, Wachtel MS, Chiriva-Internati M. Influence of obesity on the risk of developing colon cancer. Gut 2006;55:285-291.

20. Martinez-Useros J, Garcia-Foncillas J. Obesity and colorectal cancer: molecular features of adipose tissue. J Transl Med 2016;14:21.

21. Giovannucci E. Insulin, insulin-like growth factors and colon cancer: a review of the evidence. J Nutr 2001;131(11 Suppl):3109S-3120S.

22. Trabulo D, Ribeiro S, Martins C, et al. Metabolic syndrome and colorectal neoplasms: an ominous association. World J Gastroenterol 2015;21:5320-5327.

23. Ho GY, Wang T, Gunter MJ, et al. Adipokines linking obesity with colorectal cancer risk in postmenopausal women. Cancer Res 2012;72:3029-3037.

24. Huang XF, Chen JZ. Obesity, the PI3K/Akt signal pathway and colon cancer. Obes Rev 2009;10:610-616.

25. Hursting SD, Hursting MJ. Growth signals, inflammation, and vascular perturbations: mechanistic links between obesity, metabolic syndrome, and cancer. Arterioscler Thromb Vasc Biol 2012;32:1766-1770.

26. Yang HJ, Choi S, Park SK, et al. Derivation and validation of a risk scoring model to predict advanced colorectal neoplasm in adults of all ages. J Gastroenterol Hepatol 2017;32:1328-1335. 